\title{
Simulation of vibrations of a continuously elastic supported rod with varying boundary conditions under the action of a movable
}

\author{
Sergey Gridnev ${ }^{1, *}$, Yuriy Skalko ${ }^{2}$, Ilya Ravodin ${ }^{1}$, and Victoria Yanaeva ${ }^{2}$ \\ ${ }^{1}$ VSTU, Structural Mechanics Department, 394026, Voronezh, Russia \\ ${ }^{2}$ MIPT, Computational Mechanics Department, 115184, Moscow, Russia
}

\begin{abstract}
To simulate the non-linear vibrations of a floating bridge of a continuous system on separate floating supports with additional limiting supports at the ends with a moving load solves the most complicated problem which is the problem of describing the behavior of a span structure. A technique for simulating the vibration of an elastically supported deformable rod with limiting supports at the ends, which is a design scheme of a span structure, under the action of a moving force is developed. A computational algorithm for solving partial differential equations with varying boundary conditions is proposed, which includes boundary conditions in the model equations and does not require the subordination of basis functions to the boundary conditions. During the calculation, the basis remains constant. Piecewise linear basis functions are used to solve the differential equation. The technique is tested using a computational program Matlab, which is implemented when performing numerical studies of the behavior of the dynamic system as a function of the parameter changes. The developed technique is universal for studying the dynamics of a number of constructively non-linear systems.
\end{abstract}

\section{Introduction}

Simulating the vibrations of dynamic systems with the presence of constructive nonlinearity in the work with a mobile load is a very difficult task. Such systems include structures with switching on and off from work connections or having various stop limiters. In the mathematical model, differential equations in partial derivatives with varying boundary conditions are used to describe the behavior of such systems. To this class of constructions, it is possible to carry the span structures of the floating bridges of continuous system with additional restrictive rigid supports. When moving the movable load on the floating bridges of such a system, the ends of the span structure are supported by restrictive rigid supports after exhaustion of the vertical gap between the span structure and the rigid support is present (Figure 1).

\footnotetext{
*Corresponding author: gridnev_s_y@_rambler.ru
} 


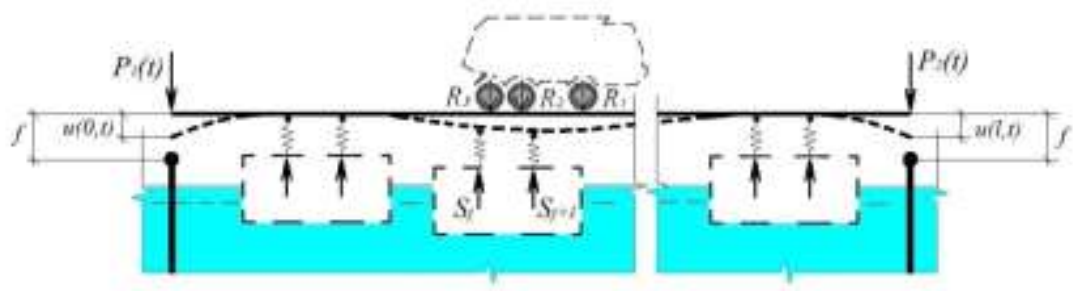

Fig. 1. Design scheme of the superstructure with restrictive rigid supports at the ends as part of the General oscillatory system «Floating bridge of continuous system+Mobile load»

The design scheme of an elastically supported span structure is a thin-walled rod of the full length supported by floating struts through elastic bonds of great rigidity, which ensures the distribution of forces from the span structure to the floating supports through the linings [1].

\section{Creating a mathematical model of the motion of a bridge}

The movement of the rod is the sum of the following motions: the motion of the center of mass of the rod, the rotation around the center of mass as a rigid body, the torsion-bending movements of the rod. A complete mathematical model must include the equations of motion for each of these components. A mathematical model for describing the complete motion of a rod is also described in detail in [1].

\subsection{Simulation of "point" forces applied to the bridge}

External forces on the side of the supports or the active load convey the impact to the rod through the contact surfaces of the car's wheels with the bridge or the contact surface of the supports with the bridge. The area of these contact surfaces is negligible compared to the characteristic dimensions of the problem and it would be desirable to formulate a mathematical model in terms of the impacts applied at a point. Doing this by limiting the use of ordinary functions is not possible. A mathematical model with "point" effects will be proposed in the work. This formulates a mathematical model in terms of generalized functions.

\subsection{Numerical solution of the constructed mathematical model}

In formulating a computational algorithm, one or another modification of the classical Galerkin method is often used. Additional difficulties in the implementation of this method for the model in question are due to the fact that supports are located at the edges of the bridge. In the free position, without load, there is a small gap between the bridge and these supports and so-called "free boundary conditions" are realized at the edge of the bridge. When the bridge is loaded, it moves and at some point rests on the support. Then, at this end of the bridge, the conditions of the "fixed boundary" are realized. That is, a one-stage change in the boundary conditions occurs. In formulating the Galerkin method, the solution is broken down into basis and the basis functions must obey the boundary conditions. That is, in the process of solving model equations, it is necessary to change the basis functions depending on the support of the ends of the rod on the limiting supports. Attempts to implement such an algorithm lead to the appearance of computational instability and require the organization of refining iterative procedures for obtaining solutions and large areas of discrepancy [2]. The same kind of difficulties arises in the construction of finite- 
difference computational algorithms [3]. Usually this problem is solved by such an artificial method as the replacement of extreme supports in the model by elastic supports with a very high stiffness coefficient [4]. In this paper, we used an approach to solving partial differential equations with discontinuous boundary conditions by reducing them to a system of ordinary equations with the help of Legendre polynomials. This made it possible to obtain a stable solution with high rigidity of the elastic supports in a wide range. Such a solution is not always satisfactory, because it does not correspond to the physical processes that take place. Also, the appearance in the model of elastic bonds with very high rigidity leads to a significant increase in requirements for a step in time to ensure computational stability. An algorithm is proposed that includes boundary conditions in the equations of the model and does not require the subordination of the basis functions to the boundary conditions and, consequently, there is no need to change the basis in the process of calculating the model solution. In the mathematical model, there are four derivatives with respect to spatial variables and the application of the frequently used basis of piecewise linear functions is not possible. In this article, a model is formulated for the solution of which an algorithm is implemented on piecewise linear basis functions. Examples of calculation of several scenarios for the motion of an elastically supported rod are given, both without and under the action of a moving load.

\section{Mathematical model}

As was mentioned above, as a design scheme of the span structure of a floating bridge, a thin-walled elastic rod model is adopted, which is under the action of a set of forces, including concentrated forces from dynamic tire pressures, forces in lining under floating supports, and forces of interaction with bridge transitions, changing in time.

We introduce a fixed coordinate system whose center coincides with the center of mass of the rod in the equilibrium position, the axis is directed horizontally from left to right, the axis is vertically upward. We also introduce a moving coordinate system whose center coincides with the current position of the center of mass of the rod and is rotated relative to the fixed system by an angle equal to the angle of rotation of the rod as a rigid body.

We introduce the notation: $z(t)$ - the position of the center of mass $(\mathrm{cm})$ of the bridge, $\varphi(t)$ - the angle of rotation of the bridge relative to the $\mathrm{m}$. in the counterclockwise direction, $y(t, x)$ - the flexural displacement of the bridge point with the coordinate in the moving coordinate system associated with the bridge, $u(t, x)=y(t, x)+z(t)+x \sin \varphi(t)-$ the position of the point of the bridge with the coordinate $x$ in the fixed coordinate system, $\mathrm{M}$ - the total mass of the bridge, $\Lambda$ - the moment of inertia of the bridge, $F(t, x)$ - the force density unit length, attached to the bridge.

$$
\begin{aligned}
& \mathrm{M} \frac{d^{2} z}{d t^{2}}=\int_{-\frac{l}{2}}^{\frac{l}{2}} F d x ; \Lambda \frac{d^{2} z}{d t^{2}}=\int_{-\frac{l}{2}}^{\frac{l}{2}} x F d x \\
& m \frac{\partial^{2} y}{\partial t^{2}}+\left(1+\aleph \frac{\partial}{\partial t}\right) E J \frac{\partial^{4} y}{\partial x^{4}}=F \\
& \frac{\partial^{2} u}{\partial x^{2}}\left(t, \mp \frac{l}{2}\right)=\frac{\partial^{3} u}{\partial x^{3}}\left(t, \mp \frac{l}{2}\right)=0, \\
& \text { if } u\left(t, \mp \frac{l}{2}\right) \geq \mathrm{u}^{*} ; \frac{\partial^{2} u}{\partial x^{2}}\left(t, \mp \frac{l}{2}\right)=\frac{\partial u}{\partial t}\left(t, \mp \frac{l}{2}\right)=0, \text { if } u\left(t, \mp \frac{l}{2}\right)<\mathrm{u}^{*}
\end{aligned}
$$


Combining the equation of the motion of the center of mass of the rod in a fixed coordinate system, the equation describing the rotation of the rod as a rigid body, the equation of the flexural displacements of the rod in the moving coordinate system associated with the rod, the boundary conditions and the initial conditions, we obtain a mathematical model of the motion of a thin-walled elastic rod simulating the flight the structure of a continuous floating bridge. Here and further throughout the initial conditions are zero.

$m$ - the linear density of the rod, EJ - the flexural rigidity of a thin-walled rod; $\aleph$ coefficient of internal friction. The boundary conditions at the ends of the rod correspond to the conditions of the rod with free ends, if the ends of the rod do not rest on the supports, and the conditions of the rod with fixed ends when the rod lies on the support. $\mathrm{u}^{*}-$ the coordinate of the upper part of the extreme support, in a fixed coordinate system.

The mathematical model (1), provided that the angle of rotation of the rod is small, so that $\sin \varphi \simeq \varphi$ can be substantially simplified. Indeed, in order to write out the equation for the flexural movements of a bridge in a fixed coordinate system, it is necessary to add inertia forces $-m\left(\frac{\partial^{2} z}{\partial t^{2}}+x \frac{\partial^{2} \varphi}{\partial t^{2}}\right)$ on the right side of the third equation.

$$
m \frac{\partial^{2} y}{\partial t^{2}}+\left(1+\aleph \frac{\partial}{\partial t}\right) E J \frac{\partial^{4} y}{\partial x^{4}}=F-m\left(\frac{\partial^{2} z}{\partial t^{2}}+x \frac{\partial^{2} \varphi}{\partial t^{2}}\right)
$$

When the angle is small, we obtain

$$
m \frac{\partial^{2} u}{\partial t^{2}}+\left(1+\aleph \frac{\partial}{\partial t}\right) E J \frac{\partial^{4} u}{\partial x^{4}}=F
$$

As a result, a mathematical model can be written in the form of an initial-boundary value problem for an unknown function $u(t, x)$ - that determines the total motion of the rod in a fixed coordinate system, eliminating the need to solve equations for intermediate variables $z(t), \varphi(t), y(t)$.

$$
\begin{aligned}
& m \frac{\partial^{2} u}{\partial t^{2}}+\left(1+\aleph \frac{\partial}{\partial t}\right) E J \frac{\partial^{4} u}{\partial x^{4}}=F \\
& \frac{\partial^{2} u}{\partial x^{2}}\left(t, \mp \frac{l}{2}\right)=\frac{\partial^{3} u}{\partial x^{3}}\left(t, \mp \frac{l}{2}\right)=0, \text { if } u\left(t, \mp \frac{l}{2}\right) \geq \mathrm{u}^{*} \\
& \frac{\partial^{2} u}{\partial x^{2}}\left(t, \mp \frac{l}{2}\right)=\frac{\partial u}{\partial t}\left(t, \mp \frac{l}{2}\right)=0, \text { if } u\left(t, \mp \frac{l}{2}\right)<\mathrm{u}^{*}
\end{aligned}
$$

We introduce the variables. This will allow us to write the model in the form of a system of equations with the second order of spatial variables

$$
\begin{aligned}
& \frac{\partial u_{1}}{\partial t}=u_{2} \\
& m \frac{\partial u_{2}}{\partial t}+E J \frac{\partial^{2} u_{3}}{\partial x^{2}}+\aleph E J \frac{\partial}{\partial t} \frac{\partial^{2} u_{3}}{\partial x^{2}}=F \\
& \frac{\partial u_{3}}{\partial t}=\frac{\partial^{2} u_{2}}{\partial x^{2}} \\
& u_{3}\left(t, \mp \frac{l}{2}\right)=\frac{\partial u_{3}}{\partial x}\left(t, \mp \frac{l}{2}\right)=0, \\
& \text { if } u\left(t, \mp \frac{l}{2}\right) \geq \mathrm{u}^{*} ; u_{3}\left(t, \mp \frac{l}{2}\right)=u_{2}\left(t, \mp \frac{l}{2}\right)=0, \text { if } u\left(t, \mp \frac{l}{2}\right)<\mathrm{u}^{*}
\end{aligned}
$$




\section{Mathematical model in terms of generalized functions}

The model (5) is formulated for distributed forces $F(t, x)$, applied to an elastic thin-walled rod. It would be desirable to formulate this model in terms of the forces applied at the point. This can be done using the technique of generalized functions described in [6]. Functions, $u_{1}, u_{2}, u_{3}$ considered as generalized functions from for an arbitrary infinitely differentiable trial function from the space of basic functions satisfy the equation.

$$
\left\{\begin{array}{l}
\int_{-\frac{l}{2}}^{\frac{l}{2}} \frac{\partial u_{1}}{\partial t} \psi d x-\int_{-\frac{l}{2}}^{\frac{l}{2}} u_{2} \psi d x=0 \\
m \int_{-\frac{l}{2}}^{\frac{l}{2}} \frac{\partial u_{2}}{\partial t} \psi d x-\left(1+\aleph \frac{\partial}{\partial t}\right) E J\left(\int_{-\frac{l}{2}}^{\frac{l}{2}} \frac{\partial u_{3}}{\partial x} \frac{\partial \psi}{\partial x} d x-\left.\left(u_{3} \frac{\partial \psi}{\partial x}+\theta\left(u^{*}-u_{1}\right) \frac{\partial u_{3}}{\partial x} \psi\right)\right|_{-\frac{l}{2}} ^{\frac{l}{2}}\right)=\int_{-\frac{l}{2}}^{\frac{l}{2}} F \psi d x \\
\int_{-\frac{l}{2}}^{\frac{l}{2}} \frac{\partial u_{3}}{\partial t} \psi d x+\int_{-\frac{l}{2}}^{\frac{l}{2}} \frac{\partial u_{2}}{\partial x} \frac{\partial \psi}{\partial x} d x-\left.\left(\frac{\partial u_{2}}{\partial x} \psi+\theta\left(u^{*}-u_{1}\right) u_{2} \frac{\partial \psi}{\partial x}\right)\right|_{-\frac{l}{2}} ^{\frac{l}{2}}=0
\end{array}\right.
$$

Here $\left.(\ldots)\right|_{-\frac{l}{2}} ^{\frac{l}{2}}=(\ldots)\left(t, x=\frac{l}{2}\right)-(\ldots)\left(t, x=-\frac{l}{2}\right)$. Let us prove this statement for the second equation of system (6). Indeed, by definition

$$
I=\left(m \frac{\partial u_{2}}{\partial t}+E J\left(1+\aleph \frac{\partial}{\partial t}\right) \frac{\partial^{2} u_{3}}{\partial x^{2}}, \psi(x)\right)=\int_{-\frac{l}{2}}^{\frac{l}{2}} m \frac{\partial u_{2}}{\partial t} \psi d x+E J\left(1+\aleph \frac{\partial}{\partial t}\right) \int_{-\frac{l}{2}}^{\frac{l}{2}} u_{3} \frac{\partial^{2} \psi}{\partial x^{2}} d x
$$

We integrate by parts

$$
I=m \int_{-\frac{l}{2}}^{\frac{l}{2}} \frac{\partial u_{2}}{\partial t} \psi d x-E J\left(1+\aleph \frac{\partial}{\partial t}\right) \int_{-\frac{l}{2}}^{\frac{l}{2}} \frac{\partial u_{3}}{\partial x} \frac{\partial \psi}{\partial x} d x+\left.E J\left(1+\aleph \frac{\partial}{\partial t}\right) u_{3} \frac{\partial \psi}{\partial x}\right|_{-\frac{l}{2}} ^{\frac{l}{2}}
$$

We again integrate by parts

$$
I=\int_{-\frac{l}{2}}^{\frac{l}{2}}\left(m \frac{\partial u_{2}}{\partial t}+E J\left(1+\aleph \frac{\partial}{\partial t}\right) \frac{\partial^{2} u_{3}}{\partial x^{2}}\right) \psi d x+\left.E J\left(1+\aleph \frac{\partial}{\partial t}\right)\left(u_{3} \frac{\partial \psi}{\partial x}-\frac{\partial u_{3}}{\partial x} \psi\right)\right|_{-\frac{l}{2}} ^{\frac{l}{2}}
$$

Given that $u_{1}, u_{2}, u_{3}$ satisfies the equations and the boundary conditions of the model (5), we obtain

$$
I=\int_{-\frac{l}{2}}^{\frac{l}{2}} F \psi d x-\left.E J\left(1+\aleph \frac{\partial}{\partial t}\right)\left(\theta\left(u^{*}-u_{1}\right) \frac{\partial u_{3}}{\partial x} \psi\right)\right|_{-\frac{l}{2}} ^{\frac{l}{2}}
$$

Comparing (8) and (10), we obtain the second equation of the system of equations (6). Similarly, we obtain other equations of the system (6).

We consider the right-hand side of the second equation of system (6). Let $F(t, x)$ corresponds to the density of forces acting on the rod on the side of the support and $F^{*}=\int_{-\frac{l}{2}}^{\frac{l}{2}} F(t, x) d x$ - the total force acting on the rod on the side of this support. The function $F(t, x)$ is equal to zero everywhere except in the region $\left(x^{*}-\Delta x, x^{*}+\Delta x\right)$ points $x^{*}$ 
corresponding to the location of this support. Then $\int_{-\frac{l}{2}}^{\frac{l}{2}} F \psi d x=\int_{x^{*}-\Delta x}^{x^{*}+\Delta x} F \psi d x$. Passing to the limit in this expression for $\Delta x \rightarrow 0$ such that $\int_{x^{*}-\Delta x}^{x^{*}+\Delta x} F d x$ remained unchanged, we obtain $\int_{-\frac{l}{2}}^{\frac{l}{2}} F \psi d x=F^{*} \psi\left(x^{*}\right)=\int_{-\frac{l}{2}}^{\frac{l}{2}} F^{*} \delta\left(x-x^{*}\right) \psi d x$. Thus, in the model (6), the concentrated forces are modeled with the help of $\delta$ - functions whose amplitude is equal to the magnitude of the acting force.

Thus, the system of equations (6), taking into account the possibility of describing the concentrated forces, is a mathematical model of the motion of the span structure of a floating bridge. The problem is to find plain functions $u_{1}, u_{2}, u_{3}$, which satisfy the model equations for an arbitrary finite infinitely differentiate test function $\psi(x) \in D$ from the space of basic functions. Note that there are no requirements for the boundary conditions on the function $u_{1}, u_{2}, u_{3}$ not superimposed. The boundary conditions have already been taken into account in the equations of the model (6).

The system of equations (6) for generalized functions $u_{1}, u_{2}, u_{3}$ and we take it as a basis for creating a computational algorithm.

\section{Computational algorithm}

The computational algorithm is based on the system of equations (6) and resembles the usual Galerkin method. The essential difference is that the basic functions over which the solution is broken down need not be subordinated to boundary conditions.

We create on the interval $\left[-\frac{l}{2}, \frac{l}{2}\right]$ mesh nodes $x_{p}, p=1 \div P$, so that $x_{1}=-\frac{l}{2}, x_{P}=\frac{l}{2}$. The grid can be uniform or uneven. This grid breaks the segment $\left[-\frac{l}{2}, \frac{l}{2}\right]$ into intervals. We connect with each node $x_{p}$ function $H_{p}(x)$, that is equal to 1 at the point $x_{p}$, equal to all other points of the grid and linear in each interval.

We define the vector function $u^{n}=\left(u_{1}, u_{2}, u_{3}\right)^{T}$. We seek the solution in the form $u^{n}(t, x)=\sum_{p} H_{p}(x) T^{p, n}(t)=H_{p} T^{p, n}$. In the last equality we used the summation convention: if in the expression the index occurs twice, then we mean the summation over all values of this index. As trial functions, we take the same piecewise linear functions numbered by the index $p w=1 \div P$. $p w$ - index, designated by two letters for convenience. Substituting these expressions in (6), we obtain, similarly to the Galerkin method, for unknown functions $T^{p, n}$ The Cauchy problem for the system of Ordinary Differential equation (ODE)

$$
\begin{aligned}
& D_{p, n}^{p w, n w} \frac{\partial T^{p, n}}{\partial t}+B_{p, n}^{p w, n w} T^{p, n}=f^{p w, n w} \\
& T^{p, n}(t=0)=0
\end{aligned}
$$

Solving this Cauchy problem for the system ODE, we find the solution $u^{n}(t, x)=\sum H_{p}(x) T^{p, n}(t)$. 


\section{Numerical experiments}

The constructed computational model was used to simulate various scenarios of the motion of a mobile force along an elastically supported rod with different parameters of the vibrating system (Figure 2).

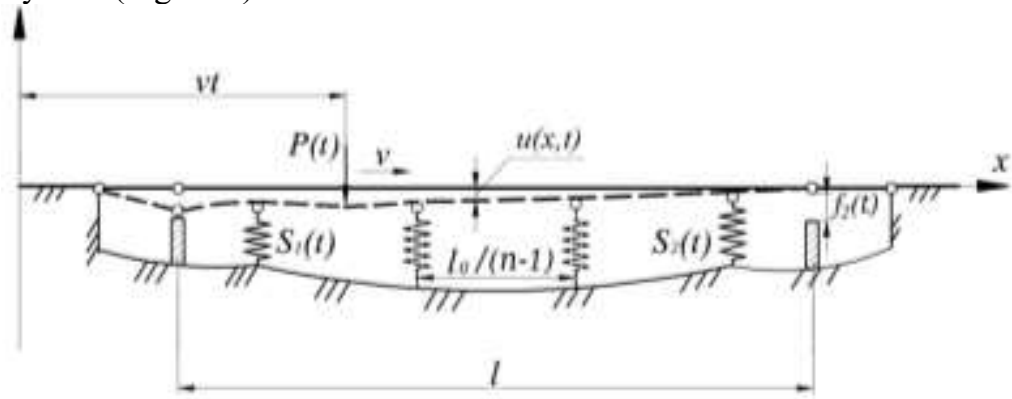

Fig. 2. Simplified design of an elastically supported deformable vibrating system for performing numerical studies (built by author)

For testing, debugging and revealing the regularities of the nonlinear behavior of an elastically supported system, we use a simplified design scheme, which is described in detail in [5]. These investigations continue in [7], where the behavior of a non-deformable elastically supported structural-nonlinear vibrating system under the action of a moving load was analyzed. Studies were carried out using a program written in Matlab software. Calculations have been made to study the effect of vibrations of an elastically supported system as a function of the gap between the limiting supports and the ends of the rod, the speed of movement of the load, and the step of sampling the rod by piecewise linear functions for a rod of length $\ell=42,5 \mathrm{~m}$, linear mass $0.888 \mathrm{~T} / \mathrm{m}$, moment of inertia of the cross section $J=0.0050936 \mathrm{~m}^{4}$, based on five elastic bonds in steps of $9,8 \mathrm{~m}$.

To illustrate some of the results of numerical studies in Figure 3 shows the deformed form of the rod when using for the solution of the initial equations of piecewise linear functions with different discretization step. The movement of the load was carried out at a speed of $15 \mathrm{~km} / \mathrm{h}$, gap was $f_{1,2}=0,15 \mathrm{~m}$, the cargo was at a distance $8,64 \mathrm{~m}$ from the left end of the rod.

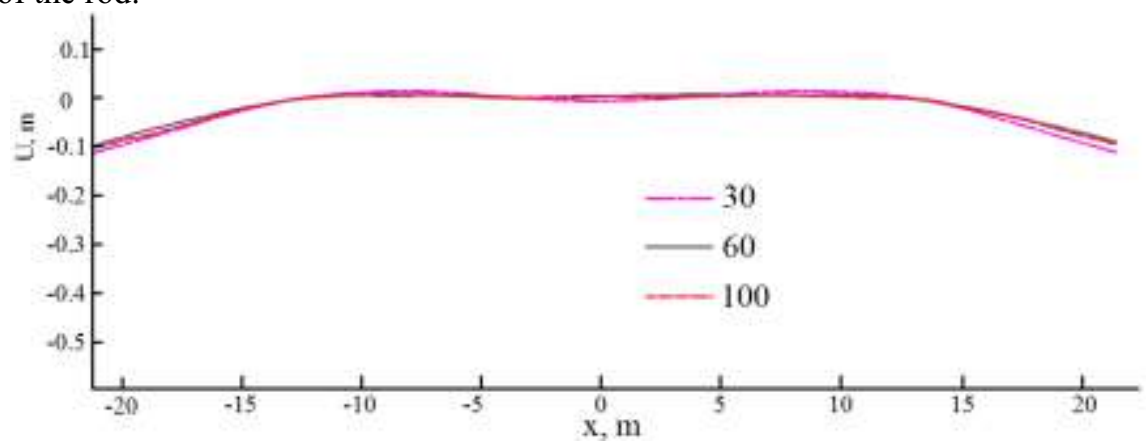

Fig. 3. The deformed form of the rod when in used for solving the original equations of piecewise linear functions with different discretization step (built by the authors)

Figure 4 shows a deformed view of the rod for comparison when using the piecewise linear functions, Chebyshev polynomials and Legendre polynomials for solving the initial equations at the time when the load was $17 \mathrm{~m}$ from the left end of the rod. 


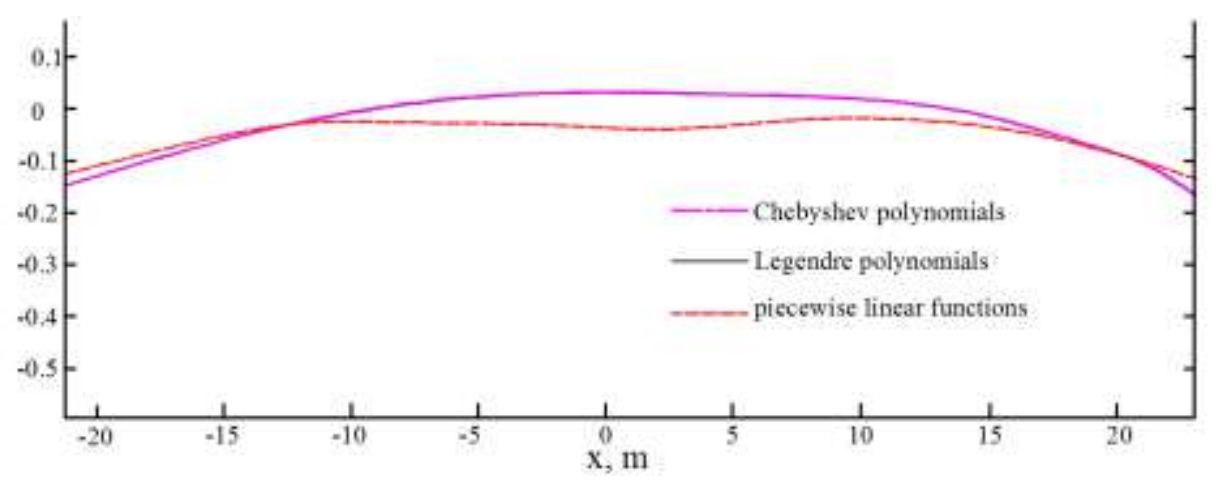

Fig. 4. The deformed form of the rod when used for solving initial equations of piecewise linear functions, Chebyshev polynomials and Legendre polynomials (built by the author)

\section{Summary and conclusion}

The technique of modeling flexural vibrations of an elastically supported beam system under a moving load is developed. For this purpose, a computational algorithm has been created for solving partial differential equations with varying boundary conditions using piecewise linear basis functions. It does not require the subordination of the basis functions to the boundary conditions. This makes it possible to obtain a stable solution by repeatedly changing the boundary conditions during the solution and to refine the local deformed state of the elastically supported system near the intermediate and restrictive rigid supports. Comparison of the obtained results with the previously obtained results using the Chebyshev and Legendre polynomials showed that the approach described above allows to adequately simulate the behavior of various constructively non-linear building structures under dynamic loading and to refine the deformed state at local sites near the supports.

\section{References}

1. S. Gridnev, Sc. Her. VSTU, Con. Arch., 9, 38-47 (2008)

2. S. Gridnev, Mat. Inter. Cong. Sc. In. Cons., 10, 83-92 (2008)

3. S. Gridnev, Privolzhsky Sc. J., 7, 64-71 (2008)

4. S. Gridnev, Sc. Her. VSTU, Con. Arch., 8, 75-83 (2014)

5. S. Gridnev, Y. Skalko, Mar. Intel. Tech., SpB 8, 37-45 (2017)

6. V. Vladimirov, Generalized functions in mathematical physics P: Science, 379 (1979)

7. S. Gridnev, Y. Skalko, I. Ravodin, V. Yanaeva, Cons. Mech. and Struc., 19, 108-117 (2017) 